\title{
EVALUACIÓN Y ANÁLISIS DEL DESARROLLO HUMANO. MENCIÓN ESPECIAL PARA ANDALUCÍA
}

Juan Carlos MORÁN ÁLVAREZ *

Pedro ÁLVAREZ MARTÍNEZ **

\section{EL MODELO DE RASCH Y EL DESARROLLO HUMANO.}

El objeto del presente estudio no es otro que presentar un nuevo enfoque y una metodología de trabajo que nos permite medir el desarrollo de los países como una variable latente o construcción teórica ${ }^{1}$, explicitada por unos items que supuestamente la definen, utilizando para ello el modelo de Rasch como instrumento de medida ${ }^{2}$.

La obtención de la medida del desarrollo, sustentada en el tratamiento de los datos, tratando de extraer de ellos la máxima información, constituye una excelente herramienta de análisis, ya que nos permite adentrarnos en la incidencia de los distintos factores en el desarrollo, así como las oportunas comparaciones y conclusiones basadas en la medición.

El enfoque tradicional en el análisis de datos se basa en el tratamiento global de los mismos, con objeto de estimar los parámetros que rigen su comportamiento. La alternativa a esta concepción, seguida en el estudio, no es más que la aplicación del ITR (Item Response Theory), la cual sugiere que si podemos modelar matemáticamente como surge un dato en función de unos parámetros, estos items apuntarán en la misma dirección del Desarrollo Humano, si bien no todos ellos se darán con la misma intensidad.

Si se obtuviera una escala que recogiera las puntuaciones de cada uno de los países, correspondiente a cada uno de los items aludidos, ¿qué país sería el más

*. Departamento de Economía Aplicada I. Universidad de Sevilla.

**. Departamento de Economía Aplicada. Universidad de Extremadura.

1. Hoi K. Suen: "Principles of Test Theories". Lawrence Earlbaum Associates, 1990.

2. Andrich, David: "Rasch Models For Measuremant". Murdoch University. Sage, 1988. 
desarrollado? Parece lógico pensar en aquel que haya obtenido mayor puntuación. No obstante, este total marginal no es una medida; los items no tienen que darse necesariamente con la misma intensidad. Es necesario conocer el grado de relevancia de cada uno de los items para obtener una medida representativa.

Ante la pregunta de qué ítem es el más relevante para el Desarrollo Humano, la respuesta dependerá de la categoría elegida. Es decir, si se calculan los correspondientes porcentajes para cada una de las categorías, por ejemplo, desarrollo débil, medio y elevado, se obtienen tres ordenaciones distintas según la clasificación obtenida para cada variable. Es necesario saber que clasificación se debe utilizar para descifrar la relevancia de cada ítem ${ }^{3}$.

Es necesario tener una clasificación de los items que intervienen en el desarrollo, que no sea ambigua; para ello se requiere un instrumento de medida que permita medir ambas cosas al mismo tiempo: el desarrollo de los países y los items que definen ese desarrollo, lo cual lleva consigo transformar los totales marginales en medida.

En los modelos de variables latentes el enfoque se centra en la interacción del sujeto con el ítem, más que en el resultado total. Este no se rechaza, pero el modelo matemático comienza modelando una respuesta a un ítem ${ }^{4}$.

El desarrollo puede ser considerado como una variable latente a lo largo del continuo donde se sitúan los parámetros $\beta_{\mathrm{n}}$, capacidad de desarrollo de un país, y $\delta_{i}$, que son los items que lo definen.

Sea la variable aleatoria $\mathrm{x}_{\mathrm{n}}$, definida como el suceso descrito por la consideración de que un país, n, esté o no desarrollado. Adoptamos el criterio de que si el país lo está, entonces su valor será 1 y en el caso contrario valor 0 ; es decir, si $\mathrm{x}_{\mathrm{ni}}=\mathrm{l}$ diremos que el país está desarrollado y si $\mathrm{x}_{\mathrm{ni}}=0$ entonces lo consideraremos como no desarrollado.

De esta forma, $\mathrm{p}\left[\mathrm{x}_{\mathrm{ni}}=1\right]$ sería la probabilidad de que el país n esté desarrollado y $\mathrm{p}\left[\mathrm{x}_{\mathrm{ni}}=0\right]$ sería la probabilidad de que el país no haya alcanzado este estado.

Los modelos de variables latentes se consideran como una dimensión única, donde se sitúan los parámetros. Para que un país n se muestre como desarrollado es necesario que su capacidad, $\beta_{n}$, pueda hacer frente a cada uno de los items, $\delta_{i}$, que lo definen, interpretándose de la forma: $\beta_{\mathrm{n}}>\delta_{\mathrm{i}}$. Así, por ejemplo, la representación gráfica ${ }^{5}$ :

3. Ellen R. Julian \& Benjamin D. Wright: "Distinguising between shared and unique employess needs". Fith International Objective Measurement Workshop. University of California, Berkeley, 1989. Mark Wilson: "Objetive Measurement. Theory into Practice". Volumen 1. Ablex Publishing Corporation, 1992.

4. E444: "Advanced Educational Measuremant. External Studes Unit. Murdoch University, Perh, Australia, 1988.

5. Wright, B. D.; Stone M. H.: "Best Test of Design". University of Chicago; Mesa press, 1979. 


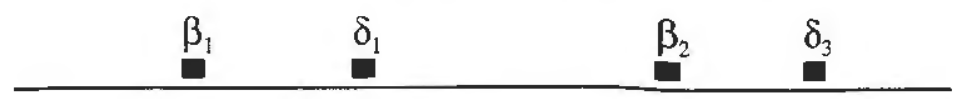

significaría que los países 2 y 3 se encuentran desarrollados respecto al ítem 1 , por ser sus parámetros $\beta_{2}$ y $\beta_{3}$ mayores que $\delta_{1}$; sin embargo, el país 1 no se considera desarrollado ya que el parámetro capacidad de desarrollo $\beta_{1}$ es inferior a $\delta_{1}$. Es decir, el ítem 1 se da en los países 2 y 3 , pero no en el 1 .

Si $\delta_{i}$ es un ítem que define el desarrollo, podemos establecer que si $\beta_{n}>\delta_{i}$, o lo que es lo mismo, si $\left(\beta_{n}-\delta_{i}\right)>0$, entonces se puede esperar que la probabilidad de que un país sea considerado como desarrollado, expresado de la forma $\mathrm{p}\left[\mathrm{x}_{\mathrm{ni}}=1\right]$, será mayor que 0,5 :

$$
\text { Si }\left(\beta_{n}-\delta_{i}\right)>0 \text { entonces } p\left[x_{n i}=1\right]>0,5
$$

Si $\beta_{\mathrm{n}}<\delta_{\mathrm{i}}$ la probabilidad de que un país se le considere desarrollado será menor que 0,5 :

$$
\text { Si }\left(\beta_{\mathrm{n}}-\delta_{\mathrm{i}}\right)<0 \text { entonces } \mathrm{p}\left[\mathrm{x}_{\mathrm{ni}}=1\right]<0,5
$$

Cuando $\beta_{n}=\delta_{i}$ ambos parámetros están situados en el mismo punto de la escala $y$, en este caso, la probabilidad de que el país sea considerado como desarrollado será de 0,5 , no pronunciándose sobre el desarrollo del país:

$$
\text { Si } \beta_{\mathrm{n}}=\delta_{\mathrm{i}} \text { entonces } \mathrm{p}\left[\mathrm{x}_{\mathrm{ni}}=1\right]=0,5
$$

Este análisis nos permite relacionar la probabilidad de que un país sea considerado desarrollado con la diferencia entre los parámetros $\beta_{\mathrm{n}}$ y $\delta_{\mathrm{i}}$. Representándola como exponente de e y con los ajustes necesarios según la literatura del modelo de Rasch se obtiene la expresión de la probabilidad de que un país sea considerado como desarrollado en función de los parámetros $\beta_{\mathrm{n}}$ y $\delta_{\mathrm{i}}$ :

$$
P\left[X_{n i}=1\right]=\frac{e^{\left(\beta_{n}-\delta_{i}\right)}}{1+e^{\left(\beta_{n}-\delta_{i}\right)}}
$$

Esta es la expresión que Georg Rasch obtuvo en el desarrollo de la variable latente ${ }^{6}$.

6. Rasch, G.: "Probabilistic Models for Some Intelligence and Attainment Test". The University of Chicago Press, 1980. 
La probabilidad de que un país no sea considerado como desarrollado será:

$$
P\left[X_{n i}=0\right]=\frac{1}{1+e^{\left(\beta_{n}-\delta_{i}\right)}}
$$

Las mejores estimaciones de $\beta_{\mathrm{n}}$ y $\delta_{\mathrm{i}}$ es la verosimilitud de la ocurrencia de los datos de la matriz $\mathrm{NxL}$ que los contiene.

$$
\lambda=\sum_{n}^{N} r_{n} \cdot \beta_{n}-\sum_{i}^{L} S_{i} \cdot \delta_{i}-\sum_{n}^{N} \sum_{i}^{L} \log _{e}\left[1+e^{\left(\beta_{n}-\delta_{i}\right)}\right]
$$

Con los cálculos apropiados se demuestra que la función de verosimilitud es máxima; para cada n se tiene

$$
r_{n}=\sum_{i=1}^{L} P\left[X_{n i}=1\right]
$$

y que para ítem

$$
S_{n}=\sum_{n=1}^{N} P\left[X_{n i}=1\right]
$$

que son las ecuaciones requeridas para la estimulación de los parámetros ${ }^{7}$.

En el caso especial que nos ocupa los datos para cada uno de los items se ha transformado en una escala de 1 a 10 , obteniendo un intervalo formado por la diferencia entre el valor máximo y el mínimo de cada ítem, dividiéndolo por diez; la asignación de categoría de 1 a 10 se realiza conforme al tramo del intervalo que le corresponde al dato.

7. E444: "Advanced Educational Measurement". Extemal Studes Unit. Murdoch University, Perth, Australia, 1988. 
Los parámetros se han obtenido mediante el método de máxima verosimilitud, utilizando las ecuaciones antes aludidas, aplicando los algoritmos PROX y UCON ${ }^{8}$ del programa Bigsteps, con 10 categorías, para lo cual se han necesitado 50 iteraciones para su convergencia ${ }^{9}$.

\section{UNA CUESTIÓN DE CUANTIFICACIÓN.}

Ante las dificultades que conlleva la medición de modelos latentes, a pesar de tener amplia información sobre los factores (items) que influyen en su constitución, comportamiento y/o evolución, en la mayor parte de las ocasiones se ha optado por la simpleza de la misma, sobre todo en los análisis basados en la Ciencia Convencional. Los defensores de una Ciencia Compleja no tratamos de negar la necesidad de buscar una representación simplificada de la realidad; es decir, un modelo que pueda configurar una representación formal de las ideas y/o conocimientos que rodean un fenómeno. No obstante, si hemos de hacer hincapié en los peligros de tratar de reducir a un sólo factor la medida de una variable latente o de condicionar la medición a la utilización de métodos estadísticos o matemáticos simples, que si bien dicen algo sobre la realidad, esta está muy alejada aún.

La medición del Desarrollo Humano ha pasado por la aceptación de alguno de estos axiomas reductores. Todo intento de no complicar las explicaciones sobre tal o cual método utilizado, ha llevado a los estudiosos del tema a huir de la falta de simplicidad, limitándose en un primer momento a la concesión en exclusividad al crecimiento económico, medido a través del PIB por habitante, de catalizador del Desarrollo, por entonces Económico. De esta forma, un país con un PIB creciente por habitante significaba automáticamente un aumento del bienestar. Sin embargo, la realidad ha mostrado Ia insensibilidad de esta medición en términos de distribución de la renta; el bienestar de una nación depende del empleo que se le haga a la renta y no de su nivel. El crecimiento de la renta es una condición necesaria -¿hasta que punto?- pero no suficiente para asegurar el desarrollo humano.

En la búsqueda de un indicador sintético, combinación de distintos indicadores en una sola medida, el Instituto de Investigación de Ias Naciones Unidas para el

8. Wright, B. D.; Masters, G.N.: "Rating Scale Analysis". University of Chicago; Mesa press, 1982.

9. Wright, B. D.; Linacre, J. M.: "A User's Guide to Bigsteps". University of Chicago. Mesa press, 1992. 
Desarrollo Social ha pasado de un desarrollo económico a un bienquisto Desarrollo Humano, donde se acentúa la importancia del concepto de pobreza en el desarrollo socioeconómico de un país. El PNUD en su "Informe Mundial sobre el Desarrollo Humano. 1990" utiliza el que denomina Indicador de Desarrollo Humano (IDH), donde vivir mucho tiempo y en buena salud, estar instruidos y tener acceso a los recursos necesarios para alcanzar un buen nivel de vida son los indicadores a sintetizar. No entramos en discusiones sobre la utilización de otros indicadores sociales que pueden influir en el desarrollo humano, y de los que se disponen datos para todos o para la mayoría de los países estudiados.

Una vez aceptada la idea de la participación de un mayor número de items en la cuantificación del Desarrollo, entramos en las carencias de las técnicas estadísticas elegidas, tal como el análisis factorial utilizando en el IDH. En palabras del informe del PNUD de 1990 "el indicador tiene el mérito de englobar las posibilidades humanas a parte de la renta, y constituye pues un paso en la dirección correcta. Por otra parte, puede ser afinado a medida que sean cuantificados un mayor número de aspectos del desarrollo humano y, por lo tanto, de las elecciones que se ofrecen a cada persona."

El éxito del indicador es indiscutible, así como esa necesidad de afinamiento del mismo que recomiendan y encargan para el futuro. Es necesario mejorar las estadísticas sobre los elementos que influyen en el Desarrollo Humano, así como homogeneizarlas para los diferentes países objeto de estudio. Al mismo tiempo hacemos nuestras las recomendaciones del PNUD sobre la necesidad de disponer de datos y analizarlos, sobre el reparto de los distintos items según los grupos de rentas; por sexo; entre regiones; diferenciando zonas urbanas y rurales; etc.

Sin embargo, es aquí donde se encuentra una segunda debilidad propia de la defensa de la simplicidad. No se va a entrar aquí en detallar el método estadístico usado por los expertos del PNUD, pues se recoge en su informe, ni se trata de negar la importancia del mismo. Solamente se pretende mostrar las diferencias que pueden surgir de la utilización de un modelo de cuantificación, basado en la consideración individualizada de los items, y no conjunta, como es propio de todo análisis factorial. Con ello se busca llamar la atención sobre los estudiosos de la materia, para conseguir el apoyo para realizar una medición más rigurosa.

El IDH se obtiene de restar a la unidad la carencia media de los tres items utilizados. La carencia de cada ítem es resultado de la relación existente entre la diferencia que un país tiene para el ítem en concreto con el mayor valor del ítem y la mayor diferencia obtenida para el conjunto de países estudiados: 


$$
I_{i j}=\frac{\left(\operatorname{máx} X_{i j}-X_{i j}\right)}{\left(\operatorname{máx} X_{i j}-\min X_{i j}\right)}
$$

en donde $\mathrm{I}_{\mathrm{ij}}$ es el indicador relativo a la variable i y en el país j. La medición efectuada a través de este sistema da una aproximación bastante real sobre el Desarrollo Humano en cada uno de los 130 países estudiados. Sin embargo, la introducción de un mayor número de items no pensamos que fuese a ser tan indulgente con el análisis factorial electo. Por otra parte, la información facilitada es bastante limitada, ya que no se introducen datos sobre el distinto peso que, sin duda alguna, tiene cada uno de los factores elegidos sobre el Desarrollo Humano, y esta carencia se hace sentir, en definitiva, en la consideración igualitaria que se realiza de cada uno de los factores sobre el IDH.

\section{UNA ALTERNATIVA AL INDICADOR DE DESARROLLO HUMANO: EL M. D. H.}

A pesar de que en fechas anteriores a la elaboración del presente trabajo, disponíamos del nuevo informe del PNUD de 1991, hemos preferido efectuar nuestra primera cuantificación y análisis sobre el desarrollo, con empleo del Modelo de Rasch, eligiendo la concepción original del IDH. La razón la podemos encontrar en que este último informe introduce un nuevo ítem: Años de escolaridad media, al que se le concede un valor de un tercio de importancia, en la representación que se realiza de la variable educación como ítem del Desarrollo Humano; los dos tercios restantes los disfruta la tasa de alfabetización de adultos. De esta forma en el IDH de 1991 se sustituye el ítem tasa de alfabetización por el de nivel de instrucción ${ }^{10}$.

No estamos en contra de la introducción de nuevos items en la cuantificación del Desarrollo Humano; todo lo contrario, nuestra intención es introducir otros indicadores sociales que influyen en el desarrollo, de los que se dispongan datos, al menos para la mayor parte de los I60 países recogidos en esta ocasión.

La adjudicación de $1 / 3$ y $2 / 3$ a uno y otro indicador social como peso dentro de un ítem conjunto no parece estar basado en ningún hecho objetivo, sino más bien intuitivo. Nuestra intención es encontrar el grado de influencia que cada

10. De esta forma, el nivel de instrucción está formado por $1 / 3 \mathrm{del}$ valor del indicador "años de escolaridad media" y $2 / 3$ del valor del indicador "tasa de alfabetización de adultos". 
ítem tiene sobre el desarrollo humano, e incluso estudiar el dominio de las distintas variables que compongan cada uno de los items elegidos. De este modo, si el ítem elegido fuese la educación, podríamos estudiar la importancia en ella de la alfabetización, de los años de escolaridad, acceso a la educación superior, etc.

Dejadas estas intenciones para posteriores estudios, tratamos de presentar la Medida de Desarrollo Humano, intentando realizar un análisis en términos comparativos, con el objeto de plantear los beneficios de una mayor y más objetiva información, producidos por la aplicación del método que perseguimos implantar en este tipo de análisis.

Los resultados de la cuantificación para los 130 países y Andalucía los podemos ver en el anexo 1, donde también se refleja el indicador obtenido por el PNUD. Con el fin de poder establecer niveles de desarrollo hemos obtenido los deciles de los indicadores (IDH y MDH), calculados en partes iguales, según la diferencia existente entre el mayor y el menor valor de cada uno.

Una vez puestas las dos mediciones frente a frente, podemos ver las diferencias de ambos métodos. El PNUD hace una clasificación de los países en función del grado de Desarrollo Humano que tiene: débil, medio o alto. Al primero corresponden aquellos países con un IDH por debajo de 0,5 ; en el segundo tramo se encuentran aquellos con un IDH por encima del 0,5 y por debajo del 0,8; por último, pertenecen al nivel alto los superiores a 0,8 e inferiores de 1 (Gráfico 4). De esta forma nos encontramos los siguientes resultados:

* Un total de 44 países tienen un Desarrollo Humano Débil

* Los países de Desarrollo Humano Medio son un total de 40 Estados.

* El grupo de Desarrollo Humano Alto está compuesto por 46 países.

Si utilizamos el mismo criterio para efectuar nuestra clasificación de la cuantificación del Desarrollo Humano, calculando la diferencia entre la mayor y el menor $\mathrm{MDH}$, asignando al tramo débil el 50\% inferior, al medio aquellos que se muevan entre el 50 y el $80 \%$ de esa diferencia y al nivel alto los que superen el $80 \%$ de la misma, nos vamos a encontrar con algunas sorpresas (Gráfico 5).

Para el tramo medio de Desarrollo Humano las diferencias no son muy importantes en cifras totales. Si para el IDH se encuentran en este tramo 40 países, en el MDH nos encontramos que son 48 los que engloban sus filas. Sin embargo, analizando cuáles son los países en uno y otro indicador, observamos como 16 países que según el IDH se encontraban en estado medio entran ahora a formar parte del tramo débil. Por otro lado, 24 participantes en el nivel elevado para el IDH descienden un nivel con las medidas obtenidas en el MDH. 
Mayores son las diferencias que encontramos para el Desarrollo Humano débil como bien se pueden desprender del párafo anterior la diferencia entre el IDH J el MDH la marcan los 16 países de tramo medio según el PNUD, que para nuestrc análisis pasan a englobar las filas de los 60 países en el nivel inferior. Según e Indicador de Desarrollo Humano la cifra sólo ascendía a 44 países.

Por último, nos choca que para el Informe comparado más de la tercera part de los países que analizan (recordamos que son 130 países), 46 en total, resulter de la cuantificación con unos resultados más que sorprendentes para algunos $\mathrm{d} \epsilon$ ellos; es decir, situarse dentro de los límites del Desarrollo Humano elevado. Nc estamos hablando ya de sus estados actuales, sino del mismo momento en que se realiza el Informe Mundial; pero resulta difícil creer que países como Malasia Colombia, Jamaica, Kuwait, Venezuela, Rumanía, México, Cuba, Panamá Trinidad y Tobago, etc., puedan considerarse dentro de este nivel.

Cuando hemos aplicado la misma regla dada para el IDH en la obtención d $\epsilon$ Desarrollo Humano elevado, nos hemos encontrado que en lugar de 46, solo 2 ? países se encuentran en este nivel, coincidente prácticamente con los de mayol resultado en el IDH, a excepción de Hong Kong y Singapur que también s encuentran en este nivel para el MDH. No es que tratemos de reducir la cifra de los desarrollados a límites muy estrechos, pero es cierto que tampoco pueden entrar er sus filas algunos de esos países. La metodología clasificatoria del Informe tiende en el procedimiento seguido para la elaboración del IDH, a dividir en partes iguales el número de países que se encuentran dentro de cada uno de los tramos: nive débil un 34\%, medio un 31\% y elevado un 35\%. Incluso en el informe de 1992 cor 160 países, la aplicación de la misma regla, que concede un $41 \%$ al nivel débil, ur $30 \%$ al medio y un $29 \%$ al elevado, muestra mayores diferencias por la introducción de 30 países nuevos en el estudio que en su mayoría pertenecen sin duda alguna al tramo menor. Para el MDH los resultados son del 46, 37 y 17\% para los niveles débil, medio y elevado, respectivamente.

Parece, pues, como si se estuviera pretendiendo justificar a los países más desarrollados, aglutinando dentro de un nivel elevado a países que no lo sor tanto. No trataremos de momento realizar otras clasificaciones, ya que parect conveniente tratar de ser algo más objetivo en ellas. Adelantar solamente que s está utilizando la aplicación del análisis multivariante poder encontrar una mejor clasificación, debido a que el modelo de Rasch está más limitado a la hora de da información en este sentido.

El siguiente paso a seguir sería analizar aquellos países que presentan claros desajustes; es decir, aquellos que muestran unas mediciones sesgadas por li contestación en alguno de sus items, no correspondida con las otras respuestas La contestación afirmativa cuando ha de ser negativa y viceversa; en este caso 
la respuesta en favor de una categoría mayor o menor cuando debería de haber sido menor o mayor, respectivamente. En este sentido encontramos un total de ocho mediciones desajustadas.

Por ejemplo, algunos de los países árabes, presentan un elevado PIB per cápita, como Omán, Arabia Saudita y otros, motivado por los recursos petrolíferos que poseen. Por lo tanto, los datos en renta no se corresponde en absoluto con la línea marcada por los otros items.

\section{RESPUESTA A LA IMPORTANCIA DE CADA ÍTEM EN EL DESARROLLO HUMANO.}

No tratamos de lanzarnos en embestida contra molinos de viento, haciendo oídos sordos a la larga ristra de recomendaciones y comentarios sobre la inexistencia de los supuestos gigantes. Evitamos aquí entrar en teorizar con profundidad el sentido del desarrollo, o del subdesarrollo, limitándonos en este caso, solamente al análisis de las respuestas obtenidas por los distintos países a través de las cifras a que ascienden los indicadores elegidos. Y lo hacemos no por falta de intenciones, sino por haber partido de la necesidad de considerar otras variables que influyen claramente en el desarrollo. Ante este impedimento solamente podemos dedicarnos al tiro de ballesta, intentando hacer blanco en la medida de nuestras posibilidades.

La elección de definición y de método de medición del desarrollo va a influir enormemente en las medidas a tomar. Es necesario tratar de depurar tanto la conceptualización como la cuantificación, de ahí que deba revisarse cada año.

El primer paso ha sido el rechazo de la consideración de la renta como medida del desarrollo, instalada en el centro de la cuestión durante las décadas de los cincuenta y los sesenta. Verdad es que el crecimiento el PNB es indispensable para atender gran parte de las necesidades humanas, al menos las esenciales a la vida humana, pero esto no hace más que confirmar su apreciación como medio para el desarrollo, en lugar de un fin en sí mismo. En ningún momento debe de confundirse el crecimiento del capital con desarrollo, situándose al Hombre como centro del mismo, atendido con el aumento de las oportunidades para mejorar el bienestar del mismo.

Hoy está claro que sociedades que han alcanzado un crecimiento económico elevado, tal es el caso de algunos países con cierto desajuste al modelo empleado, como Arabia Saudita, Emiratos Árabes, Omán, etc., sin embargo, siguen situándose en niveles bajos de desarrollo. Clara oposición es la de países que han alcanzado niveles de desarrollo superiores a los que, echando una primera mirada 
a la renta, podría esperarse, si bien este fenómeno se da en menor intensidad tales son los casos de Sri Lanka, Jamaica, Rumania, etc.

Aclarada la ruptura con la consideración de exclusividad concedida a la rent durante tanto tiempo, los estudiosos del tema se han lanzado a la búsqueda di aquellos factores que muestran cual es el grado de desarrollo de las naciones El PNUD se decide a definirlo "como el proceso de ensanche de las posibilidade afectadas a los individuos: vivir más y con buena salud, estar instruidos disponer de recursos que permitan un nivel de vida conveniente son exigencia fundamentales... El proceso de desarrollo debería crear al menos un entornı favorable que dé a los individuos y a las colectividades una posibilidad $d$ realizar sus potencialidades y de llevar una vida creativa y productiva conform a sus necesidades y a sus intereses". El centro del Desarrollo es el individu como componente de la sociedad y se obtiene a través del desarrollo de la aptitudes de los mismos.

Ante la imposibilidad de la renta de medir el desarrollo, aparecen distinta combinaciones de los distintos indicadores en una sola medida, en analogía col la renta. El Indicador Desarrollo Humano presentado por el PNUD es u ejemplo de indicador sintético que combina varios indicadores, propio de rechazo de la idea de Desarrollo Económico frente a un Desarrollo Socio-econó mico, es decir, el Desarrollo Humano.

Ya hemos habIado sobre algunas de las limitaciones del IDH; en este punt queremos hacer hincapié en una de ellas; a saber, la imposibilidad de est sinopsis por mostrar la importancia relativa de cada uno de los indicadores sociales o económicos, elegidos para el Desarrollo Humano. Es decir, no sab mostrar si es la renta, o el alfabetismo, o la esperanza de vida, el factor má importante o el que menos; la medida en que se diferencian los unos de los otros Esta diferencia de influjo existe realmente, y su importancia reside en la necesi dad de trasladar estos dominios a las mediciones de Desarrollo Humano par cada país.

La aplicación hecha de Rasch al Desarrollo Humano permite solventar est carencia, señalando la importancia de cada uno en el mismo, dando meno puntuación el ítem que más importancia tiene en el Desarrollo. Así, tenemos que del análisis conjunto de los 130 países más Andalucía, la tasa de alfabetizació es el ítem más importante a la hora de alcanzar un mejor nivel de progreso, co un total de 46,6 unidades. Le sigue la esperanza de vida al nacer con 51, unidades y, por último, el PIB real ajustado con 52,3 unidades.

Son muchos los defensores de la mayor importancia relativa de la educació y la sanidad en el Desarrollo, pero hasta ahora no había podido constatars cuantitativamente, ni siempre se refieren al mismo orden. Amartya K. Sen, pc 
ejemplo, busca la desigualdad en la educación, en la salud, en los servicios públicos, en la estabilidad de la sociedad, en el orden, . . .; sin embargo, no puede justificarlo más que en forma de reflexión o de mero ejercicio de introspectiva, incluso empapado de la experiencia y la observación.

La necesidad de una cuantificación viene de la mano de la diversidad de teorías que se pueden encontrar sobre un mismo tema. A quién creer, a quién seguir. La verdad es que sobre una misma cuestión surgen tantas doctrinas como posibles combinaciones de los distintos elementos que la configuran. Si bien existía un rechazo de la renta como principal elemento del Desarrollo, era necesario también una constatación.

Una vez cuantificado el desarrollo y obtenida la relevancia de cada ítem en esta medida, las políticas a llevar a cabo estarán más defendidas, serán más precisas. Si la elección de las medidas de Política Económica a seguir varían según se tome una definición u otra, un método y otro de cuantifícación; definido el Desarrollo Humano en función de estos tres ítem y tenida cuenta de la fineza de los resultados conseguidos con la cuantificación a través de Rasch, las alternativas a seguir serán más exactas; más eficaces y eficientes.

La importancia de este echo es innegable, evidente. Decir, basándonos en las contestaciones de los distintos países (incluyendo a Andalucía), que la alfabetización de la población, indicador que representa a la educación en el Desarrollo Humano, es la principal variable a tener en cuenta, es conceder un mayor peso a la UNESCO en las políticas de ayudas a los países del Tercer Mundo.

Conceder ayudas en términos de renta es correr el riesgo de que esa renta no sea distribuida de manera conveniente en los países receptores. De acuerdo en que las ayudas a planes nacionales de desarrollo son importantes, pero estamos hablando más bien de planes que tratan de impulsar el crecimiento económico de las naciones.

Se acepta la necesidad de no considerar la renta como variable tasadora del Desarrollo, pero se continua con una política de impulso al crecimiento de la misma como forma de salir del subdesarrollo. Es necesario dirigir esas ayudas a verdaderos planes de desarrollo, dándole mayor importancia a la educación.

La esperanza de vida es el ítem que presenta mayor desajuste, si bien esto puede estar motivado por la distinta concepción que de la variable se hace en los distintos países; la metodología empleada en su cálculo puede dar lugar a una serie de consideraciones particulares de cada caso. Hay que tener en cuenta consideraciones particulares de cada caso. Hay que tener en cuenta que se trata de esperanzas matemáticas basadas no en lo que realmente puede vivir una persona al nacer, sino lo que vivirá según la edad con la que la población existente está falleciendo. 
La tasa de alfabetización presenta un pequeño desajuste en los infit ${ }^{11}$; e decir, que se le ha dado un valor inferior al que realmente le correspondería e función de los otros items. Esto se debe, como bien puede entenderse de 1 lectura de puntos anteriores, a que parte de los países que habíamos estudiado pc su desajuste, presentaban una debilidad en la alfabetización en función de lo datos dados para los otros items, principalmente el PIB real por habitante. S trata de Somalia, Mauritania, Omán, Arabia Saudita, Emiratos Árabes y Kuwai Otro que participa en este desajuste es la República Islámica de Irán, que al igu que los mencionados presenta un desfase de -2 en alfabetización, frente al PI] y a la esperanza de vida. Etiopía presentaba un desajuste en educación, pero er en el outfit, es decir, que daba respuesta mayor a la esperada.

Si bien entre el PIB real por habitante y la esperanza de vida, la diferencia d $\epsilon$ valor es tan solo de 1,2 unidades, la alfabetización tiene una influencia mayo] distante en 4,4 unidades respecto a su inmediato perseguidor. La respuesta e contundente.

\section{INTRODUCCIÓN DE ANDALUCÍA COMO CASO ESPECIAL.}

Hasta el momento nos hemos centrado en los detalles que muestran la utilida del modelo de Rasch en su aplicación al Desarrollo Humano. Los comentario realizados hasta el momento han excluido el caso de Andalucía, de gran impor tancia en el ámbito donde se presenta este artículo, incluyéndose dentro d análisis con el fin de realizar un estudio comparativo de esta región con $\epsilon$ conjunto de países que el PNUD atiende. Por este motivo hemos dejado a u lado su atención, para no confundir a nuestro lectores en la otra alegoría d€ estudio, la del IDH frente al MDH.

Si echamos un vistazo al anexo podemos observar los resultados que para est Autonomía hemos calculado según el IDH del PNUD y el MDH obtenido segú Rasch. Para la primera medición, Andalucía se situaría dentro del grupo que el Programa de las Naciones Unidas para el Desarrollo clasifica como d Desarrollo Humano Elevado, con el valor de 0,850, por detrás de 42 de los 13 países considerados.

Según la Medida de Desarrollo Humano, Andalucía se sitúa en el octav decil, en el grupo de Desarrollo Humano Medio, según el criterio clasificatori

11. Infit es el desfase producido cuando la respuesta dada a un factor es menor de lo que, en función de l: contestaciones a los demás items, debiera haber sido. Si la respuesta es mayor respecto a las categorías elegid: para Ios otros factores hablaremos de un desfase tipo Outfit. 
empleado por el PNUD, si bien se sitúa en el límite superior de este nivel. El valor obtenido por esta Comunidad en el cálculo del $\mathrm{MDH}$ es de 64,3 unidades, compartiendo la misma medida con Grecia y la antigua República Democrática Alemana. Por delante se encuentran los 22 países que conforman el tramo de Desarrollo Humano Elevado para la medida obtenida a través del modelo de Rasch.

Andalucía se encuentra pues por encima de 18 países que según los resultados en la cuantificación del IDH para la región española, la sitúa por detrás de este listado de naciones. Se trata de Chile, las recientemente dividas Checoslovaquia y URSS, Bulgaria, Costa Rica, Uruguay, Hungría, Yugoslavia, Argentina, Polonia, República de Corea, Portugal, Trinidad y Tobago, Panamá, Cuba, México, Rumanía y Venezuela. La diferencia hallada es obvia e importante.

Hay que tener en cuenta que estos resultados se basan en las contestaciones del total de regiones apreciadas. Esto va a repercutir en los resultados; sería necesario realizar un estudio aparte para los países con un nivel de desarrollo alto, con indicadores que los cuantificasen propios a este nivel alcanzado. Medir la temperatura va a depender del lugar en que realicemos la muestra y del momento en que se realiza.

De todas formas, volvemos a indicar el carácter orientativo o indicativo del presente estudio, donde se han tomado tan solo tres indicadores para definir el Desarrollo Humano, a sabiendas de las simplificación que ello significa. De ahí que los resultados que estamos obteniendo, a parte de ser la mejor información provisional disponible, nos puede ayudar a entender la situación de cada país, en espera de la introducción de los indicadores sociales ausentes.

En un ejercicio de comparación como este no podemos olvidar confrontar Andalucía con los dos entes territoriales que engullen, que en definitiva es lo que nos permite obtener un conocimiento de la situación en la que se encuentra esta Autonomía, en términos de Desarrollo Humano, con respecto al entorno en el que se encuentra.

Por un lado, la diferencia con España ${ }^{16}$ se separa en 6,5 unidades, a favor de esta. Nuestro país presenta una Medida de Desarrollo Humano de 70,8 unidades, la segunda más alta, junto con otros ocho países: Países Bajos (127), Francia (123), Dinamarca (122), Finlandia (120), Alemania (119), Bélgica (116), Estados Unidos (112), Hong Kong (108) y por detrás de tan solo cinco países: Noruega (125), Canadá (126), Suiza (128), Suecia (129) y Japón (130), por lo cual cabe señalar que se encuentra, según esta medición, y teniendo en cuenta sus limitaciones, ya explicadas, en una situación de privilegio.

16. Incluida Andalucía 
La diferencia máxima existente en el MDH es de 25 unidades, entre Níger (1 y Mali (2) y los cinco países de Cabeza. La seis unidades y media que separa a la región del país engloba a tan solo ocho países, que por lo tanto se muestra como menos desarrollado que España y más que Andalucía.

Esto muestra las diferencias existentes dentro del país, y la peor situación e la que se encuentra la región andaluza frente al total nacional. La distancia co los datos nacionales aparece además en los tres indicadores utilizados tanto e el IDH como en el MDH, si bien es en la tasa de alfabetización, ítem de mayc influencia en el Desarrollo, donde se hace mayor.

La resta en las cifras de esperanza de vida al nacer ${ }^{17}$ son muy pequeñas, 7 y 76 años para país y región, respectivamente; ni tampoco existe mucha dispar! dad en el PIB real por habitante ${ }^{18}$ con valores de 3,95 para España y 3,80 e Andalucía. La tasa de alfabetización alcanza un $88,5 \%$ en el territorio andaluz frente al 95\% logrado para el total de España, si bien este indicador tiende asimilarse a medio plazo.

Respecto al Indicador de Desarrollo Humano, España aparece por detrás d quince países, con un indicador de 0,850. Esto nos muestra a una región much más atrasada, con respecto a la unidad territorial superior con la que se 1 compara y, por lo tanto, acentúa más aún las diferencias regionales.

Si calculamos la media de los países europeos, 'si bien solamente para hace un sencillo ejercicio de comparación (Luxemburgo no esta considerado en e Informe), la cifra sería de 68,3, por debajo de España, que tiene 70,8 unidades si bien se encontraría por encima del dato para Andalucía, con 64,3 unidades. La cifras muestran la mejor posición de este país con respecto a la media de lo comunitarios; no obstante, dentro de sus fronteras, Andalucía aparecería dentrı del grupo de las más atrasadas en la Comunidad Europea. Atraso respecto Europa y a España.

Según las medidas obtenidas por el IDH España se sitúa solamente po delante de Irlanda, Grecia y Portugal, en términos de Desarrollo Humano. Par el caso de Andalucía estos países también se encuentran por delante de dichi Autonomía. Sin embargo, según el MDH España está por encima de Portugal Grecia, Reino Unido, Italia e Irlanda y bajo la misma puntuación que el resto d socios comunitarios, a excepción de Luxemburgo no considerado en el estudic

17. Datos ofrecidos por el Instituto de Estadística de Andalucía, para 1987.

18. PIB real por habitante ajustado según el poder de compra estimado para España por el PNUD en e Informe Mundial de Desamollo Humano. El dato es de 1987 y se ha obtenido del Anuario Estadístico di Andalucía, de 1990. 
de la ONU. La Comunidad Autónoma estudiada supera en este caso a Portugal, y presentando la misma medida que Grecia.

El modelo muestra la buena situación en la que se encuentra la región del extremo sur de Europa en el contexto mundial, situándola por delante de todos los países del antiguo bloque del Este, de las economías latinoamericanas, etc. Sin embargo, se coloca por detrás de casi todos los países de la C.E. (Luxemburgo está fuera del estudio y Portugal está inmediatamente por detrás); de los países de la EFTA; de Canadá y USA; de los países asiáticos más desarrollados (Japón, Hong Kong y Singapur); de Israel y de los Oceánicos Nueva Zelanda y Australia.

La verdad es que en vista de los resultados, no podemos "maldecir la mala suerte", pero tampoco proclamar la buena "estrella".

\section{BIBLIOGRAFÍA.}

HOI K. SUEN (1990): Principles of Test Theories. Lawrence Earlbaum Associates ANDRICH, DAVID (1988): Rasch Models For Measuremant. Murdoch University. Sage.

ELLEN R. JULIAN \& BENJAMIN D. WRIGHT (1989): Distinguising between share and unique employee needs. Fith International Objetive Measurement Workshop. University of California, Berkeley.

MARK WILSON (1992): Objetive Measurement. Theory into Practice. Volumen 1. Ablex Publishing Corporation.

E444 (1988): Advanced Educational Measuremant. External Studes Unit. Murdocb University, Pertb, Australia.

WRIGHT, B. D. y STONE M. H. (1979): Best Test of Desing. University of Chicago. Mesa Press. RASCH, G. (1980): Probabilistic Models for Some Intelligence and Attainment Tests. The University of Chicago Press.

WRIGHT, B. D. y MASTERS, G. N. (1982): Raiting Scale Analysis. The University of Chicago. Mesa Press.

WRIGHT, B. D. y LINACRE, J. M. (1992): A User's Guide to Bigsteps. The University of Chicago. Mesa Press.

INFORME MUNDIAL SOBRE DESARROLLO HUMANO 1990 (1990): Programa de las Naciones Unidas sobre Desarrollo.

INFORME MUNDIAL SOBRE DESARROLLO HUMANO 1992 (1992): Programa de las Naciones Unidas sobre Desarrollo.

ANUARIO ESTADÍSTICO DE ANDALUCÍA (1990): Instituto Estadístico de Andalucía. Junta de Andalucía. 


\section{ANEXO}

\section{Medida del desarrollo humano.}

$1^{\circ}$ Decil del desarrollo humano.

\begin{tabular}{|l|l|l|c|}
\hline \multicolumn{1}{|c|}{ SECT. } & \multicolumn{1}{|c|}{ IDH } & \multicolumn{1}{|c|}{ SECT. } & MDH \\
\hline 1. Níger & 0,116 & 3. Burkika Faso & 30,9 \\
\hline 2. Mali & 0,143 & 1. Níger & 30,9 \\
\hline 3. Burkika Faso & 0,150 & 6. Guinea & 34,1 \\
\hline 4. Sierra Leona & 0,150 & 5. Tchad & 34,1 \\
\hline 5. Tchad & 0,157 & 4. Sierra Leona & 34,1 \\
\hline 6. Guinea & 0,162 & 2. Mali & 34,1 \\
\hline 7. Somalia & 0,200 & \multicolumn{2}{|c}{} \\
\cline { 1 - 3 } & & &
\end{tabular}

$2^{\circ}$ Decil del desarrollo humano.

\begin{tabular}{|c|c|c|c|}
\hline SECT. & IDH & SECT. & $\mathrm{MDH}$ \\
\hline 8. Maunitania & 0,208 & 10. Benin & 36,7 \\
\hline 9. Afganistán & 0,212 & 9. Afganistán & 36,7 \\
\hline 10. Benin & 0,224 & 8. Mauritania & 36,7 \\
\hline 11. Burundi & 0,235 & 7. Somalia & 36,7 \\
\hline 12. Bhoutan & 0,236 & 18. Senegal & 39,0 \\
\hline 13. Mozambique & 0,239 & 17. Nepal & 39,0 \\
\hline 14. Malawi & 0,250 & 15. Sudan & 39,0 \\
\hline 15. Sudan & 0,255 & 14. Malawi & 39,0 \\
\hline 16. R. Centroafricana & 0,258 & 13. Mozambique & 39,0 \\
\hline 17. Nepal & 0,273 & 14. Bhoutan & 39,0 \\
\hline 18. Senegal & 0,274 & 11. Burundi & 39,0 \\
\hline 19. Etiopía & 0,282 & & \\
\hline
\end{tabular}


$3^{\circ}$ Decil del desarrollo humano.

\begin{tabular}{|c|c|c|c|}
\hline SECT. & $\mathrm{IDH}$ & SECT. & $\mathrm{MDH}$ \\
\hline 20. Zaire & 0,294 & 25. Yemen, Rep. Árabe & 41,0 \\
\hline 21. Ruanda & 0,304 & 16. R. Centroaficana & 41,0 \\
\hline 22. Angola & 0,304 & 26. Liberia & 42,8 \\
\hline 23. Bangladesh & 0,318 & 24. Nigeria & 42,8 \\
\hline 24. Nigeria & 0,322 & 23. Bangladesh & 42,8 \\
\hline 25. Liberia & 0,333 & 22. Angola & 42,8 \\
\hline 26. Togo & 0,337 & 19. Etiopía & 42,8 \\
\hline 27. Uganda & 0,354 & & \\
\hline 28. Haití & 0,356 & & \\
\hline 29. Ghana & 0,360 & & \\
\hline 30. Yemen, Rep. Dem. & 0,369 & & \\
\hline
\end{tabular}


$4^{\circ}$ Decil del desarrollo humano.

\begin{tabular}{|c|c|c|c|}
\hline SECT. & IDH & SECT. & $\mathrm{MDH}$ \\
\hline 32. Costa de Marfil & 0,393 & 32. Costa de Marfil & 44,3 \\
\hline 33. Congo & 0,395 & 31. Yemen Rep. Dem. & 44,3 \\
\hline 34. Namibia & 0,404 & 30. Ghana & 44,3 \\
\hline 35. Tanzania, Rep. & 0,413 & 29. Haití & 44,3 \\
\hline 36. Pakistán & 0,423 & 27. Togo & 44,3 \\
\hline 37. India & 0,439 & 21. Ruanda & 44,3 \\
\hline 38. Madagascar & 0,340 & 20. Zaire & 44,3 \\
\hline & & 34. Namibia & 45,7 \\
\hline & & 33. Congo & 45,7 \\
\hline & & 28. Uganda & 45,7 \\
\hline & & 39. Papua N. Guinea & 47,0 \\
\hline & & 37. India & 47,0 \\
\hline & & 36. Pakistán & 47,0 \\
\hline & & 44. Marruecos & 48,2 \\
\hline & & 42. Kenya & 48,2 \\
\hline & & 41. Camerún & 48,2 \\
\hline & & 38. Madagascar & 48,2 \\
\hline & & 35. Tanzania, R. Un. & 48,2 \\
\hline
\end{tabular}


$5^{\circ}$ Decil del desarrollo humano.

\begin{tabular}{|c|c|c|c|}
\hline SECT. & $\mathrm{IDH}$ & SECT. & $\mathrm{MDH}$ \\
\hline 39. Papua N. Guinea & 0,471 & 47. Gabón & 49,3 \\
\hline 40. Kampuchea & 0,471 & 45. Egipto & 49,3 \\
\hline 41. Camerún & 0,474 & 43. Zambia & 49,3 \\
\hline 42. Kenya & 0,481 & 40. Kampuchea & 49,3 \\
\hline 43. Zambia & 0,481 & 55. Guatemala & 50,3 \\
\hline 44. Marruecos & 0,489 & 48. Omán & 50,3 \\
\hline 45. Egipto & 0,501 & 46. Laos, R. Dem. Pop & 50,3 \\
\hline 46. Laos, Rep. Dem.Pop. & 0,506 & 57. Algeria & 51,4 \\
\hline 47. Gabón & 0,525 & 53. Lesotho & 51,4 \\
\hline 48. Omán & 0,535 & 52. Zimbawe & 51,4 \\
\hline 49. Bolivia & 0,548 & 51. Honduras & 51,4 \\
\hline & & 50. Myanmar & 51,4 \\
\hline & & 49. Bolivia & 51,4 \\
\hline & & 60. Túnez & 52,4 \\
\hline & & 59. El Salvador & 52,4 \\
\hline & & 58. Botswana & 52,4 \\
\hline & & 56. Viet Nam & 52,4 \\
\hline & & 54. Indonesia & 52,4 \\
\hline
\end{tabular}


$6^{2}$ Decil del desarrollo humano.

\begin{tabular}{|c|c|c|c|}
\hline SECT. & IDH & SECT. & MDH \\
\hline 58. Botswana & 0,646 & 87. Jamaica & 57,9 \\
\hline 59. El Salvador & 0,651 & 86. Colombia & 57,9 \\
\hline 60. Túnez & 0,657 & 85. Malaysia & 57,9 \\
\hline 61. Irán, Rep. Islámica & 0,660 & 83. Sri Lanka & 57,9 \\
\hline 62. Rep. Árabe Siria & 0,691 & 81. Mauricio & 57,9 \\
\hline 63. Rep. Dominicana & 0,699 & 77. Emiratos Árabes & 57,9 \\
\hline 64. Arabia Saudita & 0,702 & 93. Panamá & 59,2 \\
\hline 65. Filipinas & 0,714 & 91. México & 59,2 \\
\hline 66. China & 0,716 & 90. Rumanía & 59,2 \\
\hline 67. Jamahiriya Árabe Libia & 0,719 & 89. Venezuela & 59,2 \\
\hline \multirow[t]{10}{*}{ 68. Africa del Sur } & 0,731 & 107. Chile & 60,7 \\
\hline & & 104. Bulgaria & 60,7 \\
\hline & & 102. Uruguay & 60,7 \\
\hline & & 101. Hungría & 60,7 \\
\hline & & 100. Yugoslavia & 60,7 \\
\hline & & 99. Argentina & 60,7 \\
\hline & & 98. Polonia & 60,7 \\
\hline & & 97. Corea, Rep. & 60,7 \\
\hline & & 94. Trinidad y Tobago & 60,7 \\
\hline & & 92. Cuba & 60,7 \\
\hline
\end{tabular}


$7^{\circ}$ Decil del desarrollo humano.

\begin{tabular}{|c|c|c|c|}
\hline SECT. & IDH & SECT. & $\mathrm{MDH}$ \\
\hline 50. Myanmar & 0,561 & 61. Irán, Rep. Islámica & 53,4 \\
\hline 51. Honduras & 0,563 & 70. Mongolia & 54,5 \\
\hline 52. Zimbawe & 0,576 & 68. Africa del Sur & 54,5 \\
\hline 53. Lesotho & 0,580 & 65. Filipinas & 54,5 \\
\hline 54. Indonesia & 0,591 & 64. Arabia Saudita & 54,5 \\
\hline 55. Guatemala & 0,592 & 63. Rep. Dominicana & 54,5 \\
\hline 56. Viet $\mathrm{Nam}$ & 0,608 & 62. Rep. Árabe Siria & 54,5 \\
\hline 57. Algeria & 0,609 & 74. Perú & 55,6 \\
\hline & & 73. Jordania & 55,6 \\
\hline & & 71. Nicaragua & 55,6 \\
\hline & & 69. Líbano & 55,6 \\
\hline & & 67. Jamahiriya Árabe Libia & 55,6 \\
\hline & & 66. China & 55,6 \\
\hline & & 84. Albania & 56,7 \\
\hline & & 82. Corea, Rep.Pop.Dem. & 56,7 \\
\hline & & 80. Brasil & 56,7 \\
\hline & & 79. Paraguay & 56,7 \\
\hline & & 78. Thaylandia & 56,7 \\
\hline & & 76. Irak & 56,7 \\
\hline & & 75. Ecuador & 56,7 \\
\hline & & 72. Turquía & 56,7 \\
\hline
\end{tabular}


$8^{\circ}$ Decil del desarrollo humano.

\begin{tabular}{|c|c|c|c|}
\hline SECT. & IDH & SECT. & $\mathrm{MDH}$ \\
\hline 69. Líbano & 0,735 & 106. Checoslovaquia & 62,3 \\
\hline 70. Mongolia & 0,737 & 105. URSS & 62,3 \\
\hline 71. Nicaragua & 0,743 & 103. Costa Rica & 62,3 \\
\hline 72. Turquía & 0,751 & 95. Portugal & 62,3 \\
\hline 73. Jordania & 0,752 & 88. Kuwait & 62,3 \\
\hline 74. Perú & 0,753 & 131. ANDALUCÍA & 64,3 \\
\hline 75. Ecuador & 0,758 & 110. Rep. Democrat. Alemana & 64,3 \\
\hline 76. Irak & 0,759 & 109. Grecia & 64,3 \\
\hline 77. Emiratos Árabes Unidos & 0,782 & & \\
\hline 78. Tailandia & 0,783 & & \\
\hline 79. Paraguay & 0,784 & & \\
\hline 80. Brasil & 0,784 & & \\
\hline 81. Mauricio & 0,788 & & \\
\hline 82. Corea, Rep.Pop.Dem. & 0,789 & & \\
\hline 83. Sri Lanka & 0,789 & & \\
\hline 84. Albania & 0,790 & & \\
\hline 85. Malasia & 0,800 & & \\
\hline 86. Colombia & 0,801 & & \\
\hline
\end{tabular}


$9^{\circ}$ Decil del desarrollo humano.

\begin{tabular}{|c|c|c|c|}
\hline SECT. & $\mathrm{IDH}$ & SECT. & $\mathrm{MDH}$ \\
\hline 87. Jamaica & 0,824 & 124. Australia & 66,8 \\
\hline 88. Kuwait & 0,839 & 121. Reino Unido & 66,8 \\
\hline 131. ANDALUCÍA & 0,850 & 118. Nueva Zelanda & 66,8 \\
\hline 89. Venezuela & 0,861 & 117. Italia & 66,8 \\
\hline 90. Rumania & 0,863 & 114. Irlanda & 66,8 \\
\hline 91. México & 0,876 & 113. Austria & 66,8 \\
\hline 92. Cuba & 0,877 & 111. Israel & 66,8 \\
\hline 93. Panamá & 0,883 & 96. Singapur & 66,8 \\
\hline 94. Trinidad y Tobago & 0,885 & & \\
\hline 95. Portugal & 0,899 & & \\
\hline 96. Singapur & 0,899 & & \\
\hline 97. Corea, Rep.Dem. & 0,903 & & \\
\hline
\end{tabular}


Evaluación y análisis del desarrollo humano. Mención. . .

$10^{\circ}$ Decil del desarrollo humano.

\begin{tabular}{|c|c|c|c|}
\hline SECT. & IDH & SECT. & MDH \\
\hline 98. Polonia & 0,910 & 127. Países Bajos & 70,8 \\
\hline 99. Argentina & 0,910 & 123. Francia & 70,8 \\
\hline 100. Yugoslavia & 0,913 & 122. Dinamarca & 70,8 \\
\hline 101. Hungría & 0,915 & 120. Finlandia & 70,8 \\
\hline 102. Uruguay & 0,916 & $\begin{array}{l}\text { 119. Alemania Rep. } \\
\text { Fed. Dem. }\end{array}$ & 70,8 \\
\hline 103. Costa Rica & 0,916 & 116. Bélgica & 70,8 \\
\hline 104. Bulgaria & 0,918 & 115. España & 70,8 \\
\hline 105. URSS & 0,920 & $\begin{array}{l}\text { 112. Estados Unidos de } \\
\text { América }\end{array}$ & 70,8 \\
\hline 106. Checoslovaquia & 0,931 & 108. Hong Kong & 70,8 \\
\hline 107. Chíle & 0,931 & 130. Japón & 74,3 \\
\hline 108. Hong Kong & 0,936 & 129. Suecia & 74,3 \\
\hline 109. Grecia & 0,949 & 128. Suiza & 74,3 \\
\hline 110. Rep. Dem. Alemania & 0,953 & 126. Canadá & 74,3 \\
\hline 111. Israel & 0,957 & 125. Noruega & 74,3 \\
\hline $\begin{array}{l}\text { 112. Estados Unidos de } \\
\text { América }\end{array}$ & 0,961 & & \\
\hline 13. Austria & 0,961 & & \\
\hline 114. Irlanda & 0,961 & & \\
\hline 115. España & 0,965 & & \\
\hline 116. Bélgica & 0,966 & & \\
\hline 117. Italia & 0,966 & & \\
\hline 118. Nueva Zelanda & 0,966 & & \\
\hline $\begin{array}{l}\text { 119. Alemania Rep.Fed. } \\
\text { Dem. }\end{array}$ & 0,967 & & \\
\hline 120. Finlandia & 0,967 & & \\
\hline
\end{tabular}

(Continua en la pág. siguiente. ..) 
(Viene de la pág anterior. .. )

Continuación del $10^{\circ}$ Decil del Desarrollo Humano

\begin{tabular}{|l|l|}
\hline \multicolumn{1}{|c|}{ 121. Reino Unido } & 0,970 \\
\hline 122. Dinamarca & 0,971 \\
\hline 123. Francia & 0,974 \\
\hline 124. Australia & 0,978 \\
\hline 125. Noruega & 0,983 \\
\hline 126. Canadá & 0,983 \\
\hline 127. Países Bajos & 0,984 \\
\hline 128. Suiza & 0,986 \\
\hline 129. Suecia & 0,987 \\
\hline 130. Japón & 0,996 \\
\hline
\end{tabular}


Evaluación y análisis del desarrollo humano. Mención. . .

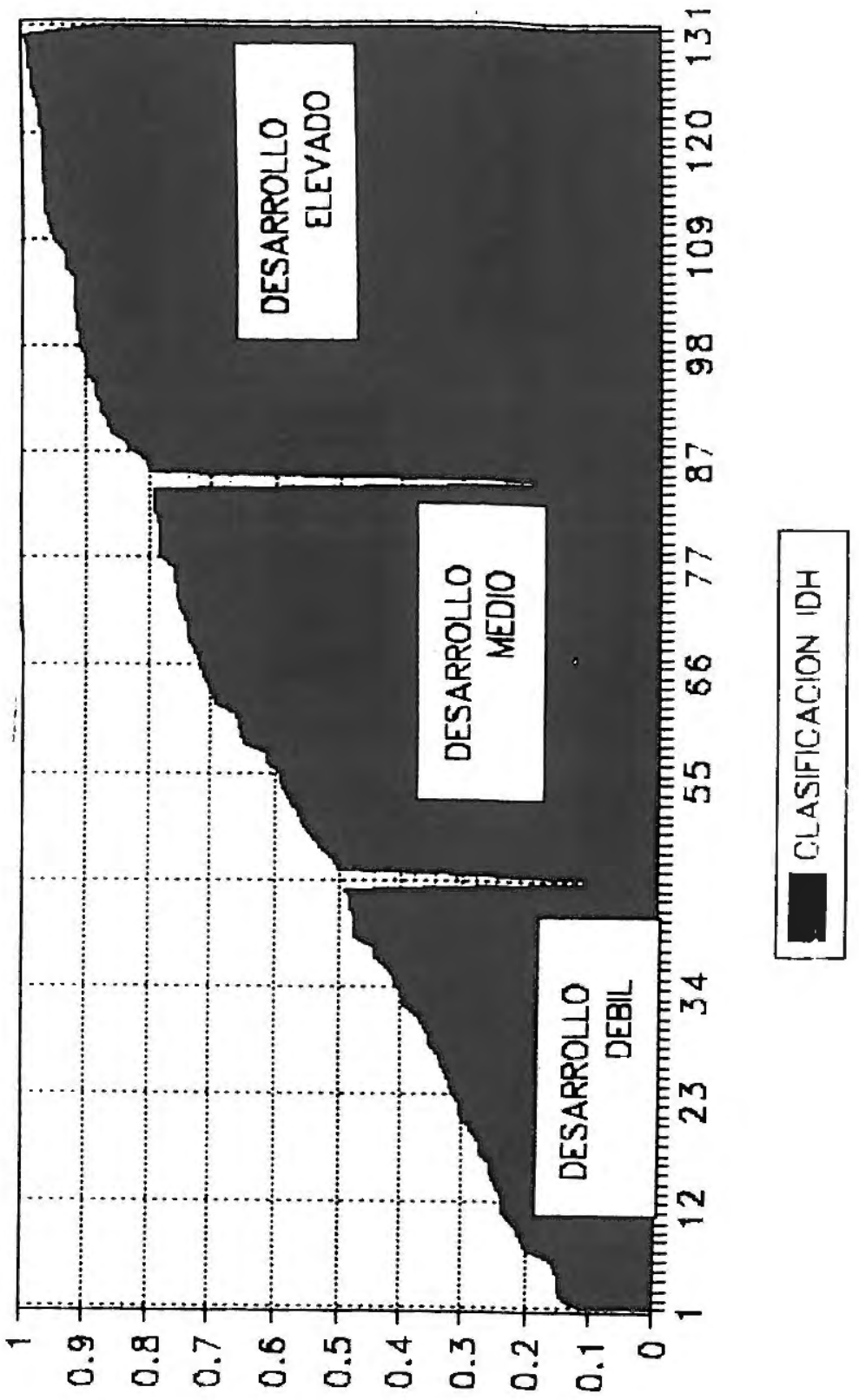




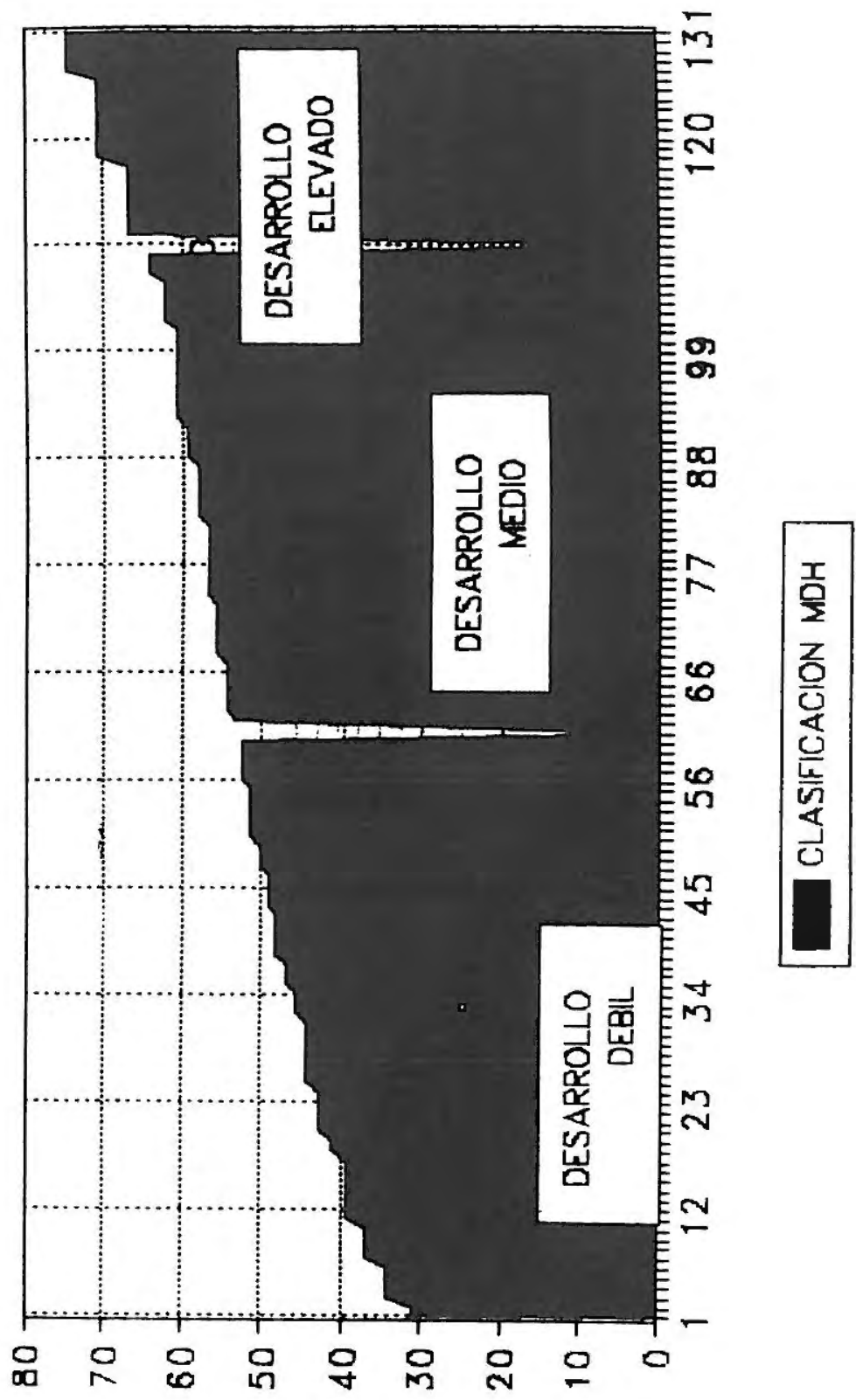

